\title{
Escrevendo resumos mais informativos
}

\author{
Débora Moura da Paixão Oliveira', Carlos Umberto Pereira² \\ Departamento de Medicina da Universidade Federal de Sergipe, Aracaju, SE \\ Núcleo de Pós-Graduação em Ciências da Saúde da Universidade Federal de Sergipe, Aracaju, SE
}

\section{RESUMO}

Objetivo: Mostrar os principais achados da pesquisa sobre resumos estruturados e discutir suas limitações, bem como fornecer aos autores inexperientes ou iniciantes as recomendações mínimas necessárias para a construção de um resumo estruturado. Método: Revisão sistemática da literatura médica sobre artigos relevantes. Resultados: Os resumos estruturados são tipicamente mais longos do que os tradicionais, mas são considerados mais informativos e acessíveis. Autores e leitores julgam ser mais úteis do que resumos tradicionais. Entretanto, existem críticas em relação ao uso de resumos estruturados e nem todos os periódicos os adotam. Conclusão: Os resumos estruturados exigem apresentação de achados de maneira sistemática, todavia são melhores que os tradicionais por serem adaptados para a maioria das investigações. A observação dos padrões dos resumos estruturados pode facilitar o processo de escrever, bem como a discussão das características dos resumos estruturados pode ser útil aos futuros autores.

\section{PALAVRA-CHAVE}

Resumos.

\section{ABSTRACT}

\section{Writing more informative abstracts}

Objective: To show the main findings from research on structured abstract and to argue its limitations, as well as to supply the unexperienced or beginning authors the minimum recommendations necessary to the construction of a structured abstract. Method: Systematic medical literature review of the relevant papers was conducted. Results: The structured abstracts are typically longer than the traditional ones, but are considered more informative and accessible. Authors and readers judge to be more useful than traditional abstracts. However, structured abstracts are not universally adopted. Conclusion: The structured format demand presentation of findings in systematic way, however they are better that the traditional ones for being adapted for the majority of the inquiries. The comment of the standards of the structured abstracts can facilitate the process to write, as well as, the characteristics of the structured abstracts will help authors of abstracts.

\section{KEY WORD}

Abstracts.

\section{Introdução}

O objetivo do pesquisador é publicar achados importantes do estudo e contribuir para avanço da ciência; no entanto, comunicar bem os resultados das pesquisas é imperativo, assim sendo, o resumo só terá impacto se os leitores puderem compreendê-lo facilmente.

O resumo é definido como a apresentação sintética dos pontos relevantes de um trabalho científico e deve refletir os dados fundamentais da investigação. ${ }^{3,17}$ O objetivo do resumo é, segundo Pereira e Oliveira, ${ }^{37}$ fornecer uma visão panorâmica do conteúdo da pesquisa. Outros autores ${ }^{8,16,33}$ acreditam que a finalidade básica deste é resumir o artigo de modo que os leitores possam decidir sobre a prioridade da leitura do artigo em questão.

Os resumos estruturados possuem forma e estrutura bem definidas; contudo, para muitos pesquisadores não é fácil escrever um resumo ou compreender inteiramente seus objetivos e finalidade. O conhecimento da estrutura básica certamente facilita o processo de escrever, apesar de que escrever bem pode ser o resultado de experiência adquirida ao longo do tempo; no entanto, a

1 Enfermeira sanitarista da Secretaria Municipal de Saúde de Aracaju, mestranda do Núcleo de Pós-Graduação em Ciências da Saúde da Universidade Federal de Sergipe, Aracaju, SE.

2 Professor doutor adjunto do Departamento de Medicina da Universidade Federal de Sergipe, neurocirurgião do Serviço de Neurocirurgia do Hospital João Alves Filho, Aracaju, SE. 
tarefa pode ser facilitada com aprendizado de algumas técnicas, paciência e dedicação., ${ }^{3,16}$

O objetivo deste artigo é mostrar os principais achados das pesquisas sobre resumos estruturados, discutir suas limitações, bem como fornecer as recomendações mínimas necessárias para a construção de um resumo estruturado aos autores inexperientes ou iniciantes.

\section{Revisão da literatura}

Segundo Alexandrov e Hennerici, ${ }^{3}$ o jeito mais eficiente de comunicar um resultado é a escolha do título e da estrutura do resumo. À exceção do título, o resumo é a parte mais lida pela maioria das pessoas. Alguns autores $^{5,16}$ o consideram a porta de entrada do estudo ou o ponto de partida da pesquisa bibliográfica.

O resumo estruturado foi inicialmente proposto, em 1987, por um grupo de pesquisadores de 18 países, incluindo o Brasil, intitulado Ad Hoc Working Group for Critical Appraisal of the Medical Literature, com a intenção de permitir, ao leitor, avaliar a validade, a importância e a aplicabilidade de um estudo., ,, $16^{-16}$

Em 1988, a Revista Paulista de Medicina propôs a redação de resumos em formato estruturado por considerá-los mais informativos. A partir de então, outras revistas começam a adotar a redação de resumos estruturados em suas publicações. ${ }^{40}$

\section{Resumo estruturado ou tradicional?}

No mundo competitivo, publicar faz a diferença e saber fazer um bom resumo é o diferencial para a publicação de um estudo., ${ }^{5,13}$ Uma vez que a estrutura do resumo é essencial, como decidir o que é melhor? Escrever um resumo estruturado ou um tradicional?

Uma pesquisa realizada por Hartley e $\mathrm{Sydes}^{24} \mathrm{com}-$ parou três pares de resumos estruturados e tradicionais. Os resultados mostraram que havia diferenças entre os três pares; os resumos estruturados eram mais longos, mais informativos e avaliados como os de melhor leitura. Os achados indicaram que os resumos estruturados eram de alta qualidade, continham mais informação e eram mais fáceis de procurar do que são os resumos produzidos de maneira tradicional.

O estudo de Sharma e Harrison ${ }^{45}$ avaliou seis jornais, três que adotavam resumos estruturados e três com resumos não estruturados, em relação ao impacto na qualidade, e concluiu que o formato estruturado possui mais qualidade de informação. O estudo corrobora com as pesquisas de vários autores. ${ }^{12,25,26,34,47}$
Uma investigação realizada no Rio de Janeiro, em 1994, analisou 68 resumos tradicionais publicados na Revista Brasileira de Enfermagem, entre 1990 e 1993 , demonstrou que a maioria dos resumos não facilitava a compreensão dos achados. Os autores, então, recomendaram a execução do modelo estruturado como forma de comunicar melhor o resultado de um estudo. ${ }^{11}$

Hartley, ${ }^{22}$ em 2004, em estudo de metanálise, comparando 31 pesquisas sobre resumos estruturados e tradicionais, mostrou que alguns investigadores acreditam que os resumos estruturados são bem aceitos por leitores e autores. ${ }^{21,26,47} \mathrm{O}$ resultado da pesquisa sugere que, para muitos autores, ${ }^{12,19,26,34,47}$ comparados com os tradicionais, resumos estruturados contêm mais informação, porém nem sempre como mostram as pesquisas de Khosrotehrani e cols. ${ }^{32}$ Para outros pesquisadores, ${ }^{19,20}$ o resumo estruturado é mais fácil de se ler e mais fácil de procurar, embora autores como Booth e O'rourke, ${ }^{4}$ assim como Wilczynski e cols., ${ }^{51}$ questionem, uma vez que, em suas pesquisas, os resumos estruturados não mostraram vantagem para a velocidade da busca. Os resumos estruturados, de acordo com alguns autores, ${ }^{18,34}$ facilitam a revisão para aceitação em congresso.

De maneira geral, a literatura é unânime em afirmar que resumos estruturados são considerados melhores para esclarecimentos sobre o método e os resultados da pesquisa do que resumos não estruturados. ${ }^{16,20,42,44}$ Entretanto, existem algumas críticas na adoção de resumos estruturados, como mostra a investigação realizada por Hartley. ${ }^{22}$ Os achados mostraram que autores ${ }^{12,20,23,28}$ consideram que resumos estruturados ocupam mais espaço e, por conterem maior número de palavras, exigem mais tempo para a leitura. Spitzer $^{46}$ acredita que o resumo estruturado exige um formato rígido, o que impede a criatividade dos autores. Outros pesquisadores descrevem que nos resumos estruturados pode haver as mesmas omissões e distorções que ocorrem em resumos tradicionais. . $^{14,20,38,39,41,43,47}$

Haynes e cols. ${ }^{27}$ descreveram que os resumos estruturados tiveram poucos erros em relação aos tradicionais, mas Pitkin e cols..$^{39}$ discutiram o reverso disso, acreditando que, por ter maior comprimento, pode haver maior número de erros. Hartley ${ }^{20}$ não encontrou nenhuma diferença nos graus de distorção ou erros entre resumos tradicionais e estruturados, mas os resumos estruturados pesquisados eram versões reescritas dos tradicionais.

Os defensores do resumo estruturado afirmam que este tem muitas vantagens: os leitores podem identificar as revisões relevantes; os vieses podem ser detectados; os resultados e as conclusões podem ser apreciados criticamente; a estrutura ajuda os autores a apresentarem seus resultados; os elementos vitais de uma revisão são 
identificados; as buscas computadorizadas de literatura são possíveis. ${ }^{12,16,19,20,26,34,42,44,47}$

\section{A confecção - Uma vez que nos decidimos pelo resumo estruturado, como confeccioná-lo?}

Segundo Nahas e Ferreira, ${ }^{35}$ o que chama mais a atenção para uma pesquisa é o título. Os autores consideram o título a maior arma para seduzir o leitor; deve refletir com precisão o conteúdo do artigo. Os leitores e os revisores verificam geralmente o título e, se interessados, leem as últimas sentenças antes que leiam o resumo completo. Alexandrov e Hennerici ${ }^{3}$ descreveram que o autor tem aproximadamente 15 segundos para convencer o leitor e, por isso, o título não deve ser excessivamente curto nem excessivamente longo. Após o título, expõem-se o nome, credenciais e local de atividade do autor. ${ }^{3,17}$

Entretanto, para outros, $, 16,33,36$ uma pesquisa começa a ser observada com origem no resumo, e a informação nele contida fará o leitor decidir-se se abandona o artigo ou o lê em profundidade. Assim sendo, ter embasamento para escrever um resumo eficientemente claro que transmita os achados de um estudo é essencial.

Atualmente, tornou-se muito importante escrever um resumo com a apresentação exata dos dados que permita aos leitores identificar rapidamente o conteúdo de um estudo e analisar se é pertinente para sua pesquisa. Os resumos sem esses dados são considerados de baixa prioridade, tendo em vista que com o crescimento da publicação on-line, na maioria das vezes, os resumos são a única parcela substantiva do artigo encontrada em muitas bases de dados eletrônicas; dessa maneira, os autores necessitam ter cuidado para que eles reflitam exatamente o conteúdo do artigo. ${ }^{8}{ }^{816} \mathrm{Em}$ geral, para assegurar-se disso, a literatura recomenda escrever o resumo após ter terminado um esboço do paper. ${ }^{33}$

O resumo estruturado deve, sobretudo, apresentar concisão e objetividade, contendo os itens necessários para que o leitor possa fazer uma avaliação da validade, da importância e da aplicabilidade: indicar os principais objetivos; descrever os métodos empregados; resumir os resultados e enunciar as principais conclusões. Atualmente, a estrutura mais usada é IMRAD (Introduction, Methods, Results And Discussion); entretanto, pode haver variações nos formatos dos resumos de diferentes periódicos, uma vez que nem todos os resumos de artigos originais são estruturados. . $^{16,17,33}$

A comunidade científica preconiza que os resumos estruturados devem conter de 150 a 200 palavras, muito embora, de maneira geral, a grande maioria contenha até 250 palavras. ${ }^{7,33}$ Entretanto, autores como Day e Gastel $^{8}$ sugerem que, se for possível expor o estudo em cem palavras, não há razão para usar 200, já que o uso de construções prolixas é inútil. O resumo deve ser tão informativo como possível sem ser demasiado longo. Um resumo muito extenso poderá ser rejeitado para uma publicação. ${ }^{1,17,27}$

\section{Introdução - 0 que é? Para quê?}

A seção de introdução deve ser a mais curta de um resumo, podendo conter somente uma única sentença. ${ }^{3,16}$ Deve ser concisa e clara, focalizando os pontos principais, empregando o menor número de palavras para exprimir o pensamento. ${ }^{37}$ Nessa seção, expõem-se os objetivos da pesquisa. Na redação dos objetivos, usa-se a forma infinitiva dos verbos. Os mais utilizados são: analisar, comparar, conhecer, correlacionar, descrever e determinar. ${ }^{17,33}$

A hipótese da pesquisa deve ser claramente indicada. ${ }^{17,37} \mathrm{Um}$ estudo realizado por De Smet e cols. ${ }^{9}$ observou que $63 \%$ dos resumos submetidos para avaliação em um congresso médico não incluíam uma hipótese específica, somente um objetivo geral. Sessenta por cento daqueles resumos contendo uma hipótese específica foram aceitos para a apresentação, dos que apresentaram somente o objetivo geral, apenas $30 \%$ foram escolhidos para apresentação.

\section{Materiais e métodos - Como?}

Uma investigação realizada por De Smet e cols. ${ }^{9}$ verificou que a falta da documentação do método de avaliação era uma deficiência principal nos resumos. A seção de materiais e métodos deve descrever o delineamento do estudo, a população, o número dos pacientes estudados (tamanho da amostra), o período da coleta dos dados e as ferramentas usadas na aquisição de dados. Os dados não devem ser informados nessa seção.

No resumo de trabalho do tipo relato de caso, as seções materiais e método são substituídas pela seção relato de caso. ${ }^{17,31,33}$

\section{Resultados - 0 que foi encontrado?}

Os resultados são a parte mais importante do resumo. Representam a solução do problema estudado, fornecem os dados que respondem à pergunta da pesquisa. Apresente os dados claramente, evite sentenças longas e 
desconcertantes, siga a lógica simples. Conduza o leitor aos achados principais. Não especule nem dê opiniões. Descreva a maioria de dados relevantes, representativos com números e estatísticas. Comece descrevendo os dados do estudo com números reais; mostre números e porcentagens absolutos; adicione os valores de p; intervalos de confiança; mencione testes estatísticos de modo que os revisores possam julgar o significado das observações. Os detalhes da análise estatística são deixados geralmente no texto propriamente dito. Evite repetir dados. ${ }^{3,33}$

A pesquisa de De Smet e cols. ${ }^{9}$ analisou resumos submetidos a um congresso de radiologia em 1993 e constatou que $50 \%$ das seções de resultados foram julgadas limitadas ou vagas. A taxa de aceitação era de $87 \%$ para os resumos contendo excelentes detalhes nessa seção e somente $7 \%$ para os resumos marcados como vagos em detalhes. O estudo constatou que o nível de detalhes na seção dos resultados é fator importante para a aceitação do resumo.

Discutir os resultados da pesquisa com os orientadores e coautores ajudará a determinar se os dados gerados foram suficientes para responder à pergunta da pesquisa no resumo. ${ }^{3,33}$

\section{Conclusões - 0 que encontramos está de acordo com os objetivos?}

A seção de conclusão deve ser destaque do resumo, o impacto da pesquisa. Deve ser uma síntese. Deve ser direta, breve, específica e seguir o objetivo da pesquisa. Frequentemente, consiste em duas sentenças. A primeira resume os achados principais, e a última indica a interpretação ou implicações clínicas. Não há necessidade de discutir ou comparar os achados com outros no resumo. $3,29,30,33$

Um erro comum de iniciantes na pesquisa é apresentar informação e conclusão no resumo que não figurem no trabalho e muitos resumos discordam com o texto do artigo. Os autores devem ter cuidado para evitar as conclusões que não são claramente embasadas pelos resultados da pesquisa. As conclusões precisam ser baseadas nesses achados. ${ }^{29,33}$

Não superestime a importância dos achados da pesquisa. Evite reivindicações mesmo em estudos pioneiros. Seja específico focalizando os achados do estudo e o que significam. ${ }^{3,30}$

Não há necessidade de dizer que uma pesquisa adicional é necessária, uma vez que os cientistas nunca param suas explorações. ${ }^{3}$

A seção de conclusão é redigida no tempo verbal presente. Se o espaço permitir, pode-se começar com uma indicação de abertura: "nosso estudo mostra..." e resume "os achados do estudo..." É recomendável usar a sentença "em conclusão". Evite as expressões: "o autor descreve..."; "o autor encontrou..."; "o autor pesquisou..."; "neste trabalho, o autor expõe..." Dê preferência a: descreve-se, expõe-se. ${ }^{17}$

\section{Seguir toda a metodologia é garantia que o trabalho será escolhido?}

Um estudo realizado em 2007 por Wenzel ${ }^{50}$ demonstrou que menos de $50 \%$ dos resumos apresentados em conferências têm seus artigos inteiramente publicados, porém as investigações de Alexandrov e Hennerici ${ }^{3}$ consideraram que esse número pode ser maior, chegando ao percentual de $60 \%$ a $75 \%$.

Uma investigação realizada por dos Santos e Pereira ${ }^{10}$ mostrou que seguir toda a metodologia não é garantia que o trabalho será escolhido. A cada ano, milhares de resumos são submetidos para apresentação em conferências e revisões para publicação, diminuindo as chances de um trabalho ser selecionado. ${ }^{3,18} \mathrm{O}$ estudo de dos Santos e Pereira ${ }^{10}$ avaliou e comparou a qualidade dos resumos apresentados no congresso da Sociedade Brasileira de Infectologia, realizado em 2005. A hipótese era que o material de melhor qualidade seria escolhido para a apresentação oral. A pesquisa comparou os 63 resumos selecionados para a apresentação oral com uma amostra aleatória $(n=63)$ dos 664 resumos registrados como apresentações em pôster. A qualidade dos resumos foi considerada de moderada a boa. A pesquisa concluiu que a hipótese testada não foi confirmada; ou seja, nem todo material de boa qualidade foi escolhido. O estudo chama a atenção dos organizadores de congressos para uso de critérios de avaliação da qualidade dos resumos.

No sentido de evitar essas distorções, Timmer e cols. ${ }^{48}$ publicaram um questionário contendo itens utilizados pelos revisores como indicador da qualidade de um resumo estruturado (Quadro 1).

Autores como Hopewell e cols. ${ }^{29}$ estudaram o CONSORT (Consolidated Standards of Reporting Trials), outro instrumento de ajuda a autores de resumos a fornecer detalhes e claridade necessários aos leitores que desejam avaliar a validade e a aplicabilidade de uma experimentação. $\mathrm{O}$ instrumento busca: critério de eleição dos pacientes, local e data da coleta; como os participantes foram alocados para a intervenção; conhecimento científico e explanação de motivos; objetivos específicos e hipóteses; como o tamanho da amostra foi determinado; se interpretação dos resultados está de acordo com a hipótese do estudo, possui bias ou imprecisão e perigo de associação com multiplicidade de análise e resultados. 


\begin{tabular}{|c|c|c|c|}
\hline \multicolumn{4}{|l|}{$\begin{array}{c}\text { Quadro } 1 \\
\text { Instrumento indicador da qualidade de um resumo estruturado }\end{array}$} \\
\hline Qualidade da Avaliação & parcialmente & não & $\mathbf{n} / \mathbf{a}$ \\
\hline \multicolumn{4}{|l|}{ A pergunta/objetivo foi descrita suficientemente? } \\
\hline \multicolumn{4}{|l|}{ Delineamento evidente e apropriado para responder à pergunta do estudo? } \\
\hline \multicolumn{4}{|l|}{ Características dos sujeitos são suficientemente descritas? } \\
\hline \multicolumn{4}{|l|}{ Sujeitos apropriados para a pergunta do estudo? } \\
\hline \multicolumn{4}{|l|}{ Controles usados e apropriados? (se não tem controle, checar não ) } \\
\hline \multicolumn{4}{|l|}{ Métodos de seleção dos sujeitos são descritos e apropriados? } \\
\hline \multicolumn{4}{|l|}{ O estudo randomizado foi possível, ele foi descrito? (se não foi possível, checar n/a) } \\
\hline \multicolumn{4}{|l|}{ Se cegar os investigadores para a intervenção foi possível, isso foi relatado? (Se não possível, n/a) } \\
\hline \multicolumn{4}{|l|}{ Se cegar os sujeitos para intervenção foi possível, isso foi relatado? (Se não possível, n/a) } \\
\hline \multicolumn{4}{|l|}{ Resultados bem-definidos e capazes de medir viés (bias)? Meios da avaliação relatados? } \\
\hline \multicolumn{4}{|l|}{ Confusão explicada? } \\
\hline \multicolumn{4}{|l|}{ Amostra com tamanho adequado? } \\
\hline \multicolumn{4}{|l|}{ Análise estatística apropriada? } \\
\hline \multicolumn{4}{|l|}{ Testes estatísticos indicados? } \\
\hline \multicolumn{4}{|l|}{ Valor de p ou intervalo de confiança indicado? } \\
\hline \multicolumn{4}{|l|}{ Resultados descritos com detalhes suficientes? } \\
\hline \multicolumn{4}{|l|}{ Os resultados suportam as conclusões? } \\
\hline Soma (itens) & & & \\
\hline
\end{tabular}

Adaptado de Timmer e cols. ${ }^{48}$

\section{Quais cuidados tomar para ter o resumo escolhido?}

O resumo é a parte fundamental do trabalho sendo o primeiro parágrafo considerado o mais impactante. Entretanto, a escolha de um título dinâmico e informativo é o que chama mais a atenção. ${ }^{17,37}$

Certifique-se de que o resumo seja conciso, porém contenha os elementos principais do artigo, reflita o conteúdo, enfatize as ideias e siga todas as regras. ${ }^{3,16,17,33,37}$

O International Committee of Medical Journal Editors $^{31}$ recentemente atualizou as exigências para resumos submetidos aos jornais biomédicos, as quais foram seguidas pela maioria dos jornais. De acordo com o comitê, um resumo deve fornecer o contexto para o estudo, indicar suas finalidades, os procedimentos básicos, os métodos, os principais achados, seu significado estatístico e as principais conclusões; deve enfatizar aspectos novos e importantes do estudo. ${ }^{16,17}$

Ter cuidado com o texto, ser claro, usar terminologia específica é importante; um texto sem pompa nem rebuscamento estimula à leitura agradável e torna-se de fácil e rápida compreensão. O resumo deve ter as qualidades essenciais do texto científico: clareza, concisão e precisão. Entretanto, para consegui-lo, é necessário rever o texto várias vezes para corrigi-lo e cortar o supérfluo. Tente evitar a voz passiva, se puder. Procure utilizar frases pouco extensas e sempre em ordem direta. ${ }^{36}$ Revisar a ortografia, bem como o texto do abstract escrito em inglês. Mostrar o esboço aos colegas para crítica é sempre útil., 3,17,36,49

Considere os regulamentos específicos feitos pelos organizadores de conferências e jornais que são diferentes no estilo e no contexto. $\mathrm{O}$ formato requerido para resumos estruturados difere de jornal a jornal, e alguns jornais usam mais de uma estrutura; os autores devem preparar seus resumos no formato especificado pelo jornal ou pelos eventos científicos que escolheram. ${ }^{16,17}$

Seguir as recomendações quanto às normas de impressão do texto é aconselhável, tipo e tamanho de fonte, tamanho do espaçamento entre as linhas, tipo de editor de texto, apresentação do título, nome e número de autores, endereço do autor principal e instituição onde o trabalho foi realizado. ${ }^{17}$

A aceitação de um resumo está basicamente condicionada à qualidade do conteúdo e à observação estrita das normas para publicação de cada revista. Não aderindo às regras para submissão de um trabalho, corre-se o risco elevado de não ser aprovado. ${ }^{3,6,17}$

Grimm, ${ }^{15}$ em 2005, comparou os critérios de revisões editor-escolhidas e autor-sugeridas; os investigadores encontraram pouca diferença na qualidade das revisões entre os dois grupos. Entretanto, as revisões autor-sugeridas eram mais prováveis à aceitação do manuscrito $(47 \%$ contra $35 \%)$ e menos prováveis à rejeição (10\% contra $23 \%)$. 
Alexandrov e Hennerici ${ }^{3}$ acreditam que selecionar com cuidado a categoria apropriada para a submissão do resumo de acordo com o tema é importante, se selecionada apropriadamente, é provável que o resumo seja classificado por pares com interesses e familiaridade similares.

\section{Considerações finais}

É recomendável que a frase inicial do resumo não repita o que está dito no título. ${ }^{3,16}$

O uso de abreviações, números, tabelas e referências bibliográficas de maneira geral não são citados no resumo exceto em casos raros. As abreviações somente devem ser usadas quando um termo se repete várias vezes. Nesse caso, a abreviatura será dada entre parênteses em sua primeira menção no texto e será utilizada a seguir. A referência bibliográfica só é citada eventualmente, quando se menciona uma modificação de método. ${ }^{8,16,17}$

Palavras-chave: Para fins de indexação, o resumo deve incluir termos representativos e palavras-chave relacionadas ao assunto. É recomendável evitar a repetição de palavras que já constem no título. Use termos utilizados em buscas bibliográficas e que podem ser importantes na localização do artigo. Devem ser mencionadas por ordem alfabética. ${ }^{7,33}$

A palavra abstract em inglês significa muito mais que apenas resumo. A palavra, dividida em sílabas, significa o AB - absolutely (absolutamente) STR - straightforward (estratégico) ACT - actual data presentation and interpretation (apresentação e interpretação de dados atuais). ${ }^{3} \mathrm{O}$ abstract não precisa ser necessariamente uma tradução literal do resumo em português, porém o tamanho, o conteúdo e as qualidade das informações devem ser as mesmas. A estrutura gramatical do inglês é distinta do português; traduções literais podem não soar bem. Evite o uso de dicionários para traduzir termos técnicos específicos; é melhor verificar em publicações de língua inglesa. ${ }^{17,49}$

A literatura sugere que os resumos estruturados são melhores que os tradicionais por serem mais informativos, facilitam a revisão por pares, podem ser adaptados para a maioria das investigações, incluindo revisões e estudos experimentais. São mais fáceis de compreender uma vez que o texto é escrito em parágrafos mais curtos, ajudam os autores a não omitir dados relevantes e revelam erros metodológicos. No entanto, o formato requer que os autores organizem e apresentem seus achados de maneira sistemática. Escrever um bom resumo requer atenção considerável aos detalhes, visto que uma boa pesquisa merece um resumo claramente escrito.
Tivemos a pretensão de fornecer as recomendações mínimas necessárias para a construção de um resumo estruturado. Esperamos que tenham sido úteis aos futuros autores. Detalhes adicionais poderão ser encontrados na literatura sobre o tema bem como em livros-textos especializados.

\section{Referências}

1. Abu-Ali AN, Hafidh JZ. How to Write an A+ Paper. ICTA. 2007;7:299.

2. Ad Hoc Working Group for Critical Appraisal of the Medical Literature. A proposal for more informative abstracts of clinical articles. Ann Intern Med. 1987;106:598-604.

3. Alexandrov AV, Hennerici MG. Writing good abstracts. Cerebrovasc Dis. 2007;23:256-9.

4. Booth A, O'Rourke AJ. The value of structured abstracts in information retrieval from MEDLINE. Health Libr Rev. 1997;14:157-66.

5. Brown JM. Driven to abstraction: writing an abstract for presentation or publication. J Intraven Nurs. 1989;12:326-8.

6. Burgdorf WH. How to get your paper accepted in an Englishlanguage journal. J Dtsch Dermatol Ges. 2004;2:592-6.

7. Cremmins E. The art of abstracting. 2.ed. Info Resources Press, April 1996.

8. Day RA, Gastel B. Cómo escribir trabajos científicos. 4.ed. Washington: PAHO, 2008.

9. De Smet AA, Manaster BJ, Murphy Jr. WA. How to write a successful abstract. Radiology. 1994;190:571-2.

10. dos Santos EF, Pereira MG. Quality of the structured abstracts presented at a congress. Rev Assoc Med Bras. 2007;53:355-9.

11. dos Santos I, Clos AC. The most informative abstracts of research papers: suggestion of a model. Rev Bras Enferm. 1996;49:135-46.

12. Dupuy A, Khosrotehrani K, Lebbe C, Rybojad M, Morel P. Quality of abstracts in 3 clinical dermatology journals. Arch Dermatol. 2003;39:589-93.

13. Elizondo-Rivera RL, Bosques-Padilla FJ. How to write a scientific article: how to get your research published. Rev Gastroenterol Mex. 2007;72:113-6.

14. Froom P, Froom J. Deficiencies in structured medical abstracts. J Clin Epidemiol. 1993;46:591-4.

15. Grimm D. Peer review. Suggesting or excluding reviewers can help get your paper published. Science. 2005;309:1974. 16. Guimarães CA. Structured abstracts: narrative review. Acta Cir Bras. 2006;21:263-8.

17. Gusmão SS, Silveira RL. Redação de resumo de tema livre para congresso médico. Arq Bras Neurocir. 2001;20:106-9.

18. Happell B. Hitting the target! A no tears approach to writing an abstract for a conference presentation. Int J Ment Health Nurs. 2007;16:447-52.

19. Hartley J. Applying ergonomics to applied argonomics: using structured abstracts. Appl Ergon. 1999;30:535-41.

20. Hartley J. Are structured abstracts more or less accurate than traditional ones? A study in the psychological literature. J Info Sci. 2000;26:273-7.

21. Hartley J. Improving the clarity of journal abstracts in psychology. Science Comm. 2003;24:366-79. 
22. Hartley J. Current findings from research on structured abstracts. J Med Libr Assoc. 2004;92:368-71.

23. Hartley J, Ganier F. Which do you prefer? Some observations on preference measures in studies of structured abstracts. Euro Sc Editing. 2000;26:4-7.

24. Hartley J, Sydes M. Are structured abstracts easier to read than traditional ones? J Res Reading. 1997;20:122-36.

25. Hartley J, Sydes M, Blurton A. Obtaining information accurately and quickly: are structured abstracts more efficient? J Info Sc. 1996;22:349-56.

26. Haynes RB. More informative abstracts: current status and evaluation. J Clin Epidemiol. 1993;46:595-7.

27. Haynes RB, Mulrow CD, Huth EJ, Altman DG, Gardner MJ. More Informative Abstracts Revisited. Ann Intern Med. 1990;113:69-76

28. Heller MB. Structured abstract: a modest dissent. J Clin Epidemiol. 1991;44:739-40.

29. Hopewell S, Clarke M, Moher D, Wager E, Middleton P, Altman DG, Schulz KF. CONSORT for Reporting Randomized Controlled Trials in Journal and Conference Abstracts: Explanation and Elaboration. PLoS Medicine. 2008;5:48-56.

30. Hoppin FG. How I review an original scientific article. Am J Resp Crit Care Med. 2002;166:1019-23.

31. International Committee of Medical Journal Editors. Uniform requirements for manuscripts submitted to biomedical journals. N Engl J Med. 1997;336:309-15.

32. Khosrotehrani K, Dupuy A, Lebbe C, Rybojad M, Morel P. Qualite des resumes des articles publies dans les Annales de Dermatologie. Ann Dermatol Venereol. 2002;129:1271-5.

33. Mezzadri JJ. Como escribir un resumen estructurado. Rev Neurocirugía. 2007;9:92-5.

34. McIntosh N, Duce G, Sedin G. Structure improves content and peer review of abstracts submitted to scientific meetings. Euro Sc Editing. 1999;25:43-7.

35. Nahas FX, Ferreira LM. Análise dos itens de um trabalho científico. Acta Cir Bras. 2005;20:13-6.

36. Neill US. How to write a scientific masterpiece. J Clin Invest. 2007;117:3599-602.

37. Pereira CU, Oliveira DMP. Produzindo um pôster científico. Arq Bras Neurocir. 2007;26:64-71.

38. Pitkin RM, Branagan MA. Can the accuracy of abstracts be improved by providing specific instructions? A randomized controlled trial. JAMA. 1998;280:267-9.

39. Pitkin RM, Branagan MA, Burmeister L. Accuracy of data in abstracts of published research articles. JAMA. 1999:281:1110-1.
40. Resumos Estruturados. Reformulação dos resumos de ensaios clínicos [editorial]. Rev Paul Med. 1988;106:183-4.

41. Salager-Meyer F. Medical English abstracts: how well are they structured? J Am Soc Inf Sci. 1991;42:528-31.

42. Santos EF, Pereira MG. Qualidade dos resumos estruturados apresentados em congresso médico. Rev Assoc Med Bras. 2007;53:355-9.

43. Scherer RW, Crawley B. Reporting of randomized clinical trial descriptors and use of structured abstracts. JAMA. 1998;280:269-72.

44. Shannon S. Writing a structured abstract. Can Assoc Radiol J. 2000:51:328-9.

45. Sharma S; Harrison JE. Structured abstracts: do they improve the quality of information in abstracts? Am J Orthod Dentofacial Orthop. 2006;130:523-30.

46. Spitzer WO. The structured sonnet. J Clin Epidemiol. 1991;44:729

47. Taddio A, Pain T, Fassos FF, Boon H, Ilersich AL, Einarson TR. Quality of nonstructured and structured abstracts of original research articles in The British Medical Journal, The Canadian Medical Association Journal and The Journal of the American Medical Association. CMAJ. 1994;150:1611-5.

48. TimmerA, Sutherland LR, Hilsden RJ. Development and evaluation of a quality score for abstracts. BMC Med Res Methodol [serial on the Internet]. 2003;3:2. Disponível em: http://www. biomedcentral.com/content/pdf/1471-2288-3-2.pdf

49. Tompson A. How to write an English medical manuscript that will be published and have impact. Surg Today. 2006;36:407-9.

50. Wenzel V, Dünser MW, Lindner KH. How do I write an original article? An introduction for beginners. Anaesthesist. 2007;56:828-36.

51. Wilczynski NL, Walker CJ, MCKibbon KA, Haynes RB. Preliminary assessment of the effect of more informative (structured) abstracts on citation retrieval from MEDLINE. MEDINFO Proceedings. 1995;8:1457-61.

Original recebido em novembro de 2009

Aceito para publicação em janeiro de 2010

Endereco para correspondência

Débora Moura da Paixão Oliveira

Av. Augusto Mavnard, 245/404

49015-380 - Aracaju, SE 